\title{
Multiple Occipital, Parietal, Temporal, and Frontal Foramina: A Variant of Enlarged Parietal Foramina in an Infant
}

\author{
Erdem Y1lmaz ${ }^{1}$, Aylin Yetim², Oğuz Bülent Erol ${ }^{3}$, Melih Pekcan $^{1}$, Ensar Yekeler ${ }^{3}$ \\ ${ }^{1}$ Department of Radiology, İstanbul University İstanbul Faculty of Medicine, İstanbul, Turkey \\ ${ }^{2}$ Department of Pediatrics, İstanbul University İstanbul Faculty of Medicine, İstanbul, Turkey \\ ${ }^{3}$ Department of Pediatric Radiology, İstanbul University İstanbul Faculty of Medicine, İstanbul, Turkey
}

Background: Calvarial lesions are often detected incidentally in routine radiology. Most lytic lesions of the skull are benign. Enlarged parietal foramina are benign lesions caused by deficient intramembranous ossification.

Case Report: An 11 month-old female patient was admitted with a mass on the right of the back of the head. Physical examination showed a soft $5 \mathrm{~cm}$ mass area with no palpable bone in the right occipital. The family history revealed a similar mass in a maternal cousin that resolved over time. Craniography showed lytic lesions, and there were no other pathologies on a complete skeletal X-ray. Computed tomography (CT) showed regular-shaped defects in the bilateral temporal bones, right parietal bone, bilateral frontal bones in the upper-medial orbital wall, and particularly in the occipital bone. The well-defined contours, absence of a soft tissue component, and normal structure and density of the adjacent calvarial bones all pointed to a congenital defect. No change in the lesions was observed during a three-year ultrasound follow-up period.

Conclusion: To the best of our knowledge, this is the first described case of multiple occipital, parietal, temporal, and frontal foramina in the cranium. A diagnosis of enlarged parietal foramina variant should be considered after ruling out the differential diagnosis in patients with multiple calvarial lesions. CT may provide valuable findings for the differential diagnosis, and sonography may be used for follow-up.

Key Words: Computed tomography, occipital foramina, parietal foramina
Calvarial lesions are often detected incidentally in radiological studies (1). The frequency of suspicious calvarial lytic lesions found on radiographs is approximately $7 \%$ (2). Many developmental disorders affect the parietal bones (3). The prevalence of enlarged parietal foramina is less than 1 in 25,000 individuals (4). Although craniography provides important information, its use has been decreasing since the development of computed tomography (CT). CT allows for a more detailed and accurate examination of the skull, as well as evaluation of intracranial structures in three-dimensional (3D) format images (5). Here, we describe an 11 month-old female patient that presented with a mass in the occipital area that was shown to have multiple calvarial defects (a variant of enlarged parietal foramina) by further testing. To the best of our knowledge, this is the first reported case of multiple parietal, occipital, temporal, and frontal foramina.

\section{CASE PRESENTATION}

An 11 month-old female patient presenting with a mass on the right side of the back of the head applied to our center. There had been no previous complaints, but the $5 \times 5 \mathrm{~cm}$ mass was noticed at seven months of age. The patient history was unremarkable. A family history revealed that a similar mass was found and eventually resolved in the patient's maternal cousin. Oral informed consent was obtained from the patients' parents. A physical examination showed normal neurological and physical development, and the anterior fontanelle was open and the posterior was closed. A $5 \mathrm{~cm}$ soft mass with no palpable bone tissue was palpated on the right side of the back of the head. The complete blood count and biochemistry were unremarkable, and thyroid and parathyroid hormones were normal. A craniography showed lytic lesions, and no pathology was detected on the skeletal X-ray. A magnetic resonance 


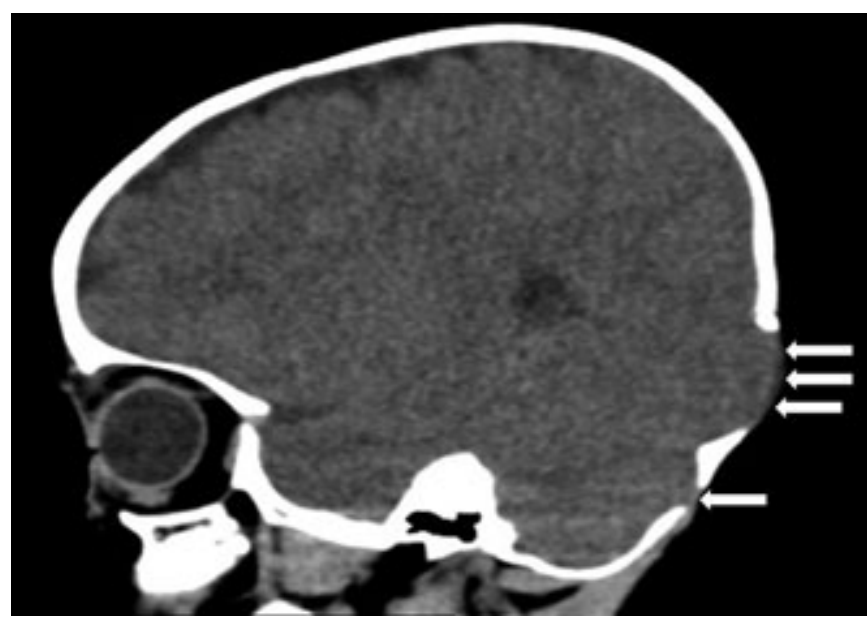

FIG. 1. A sagittal CT scan showed a soft tissue mass-mimicking slight swelling (arrows) and lytic lesions on the right side of occipital bone during the physical examination

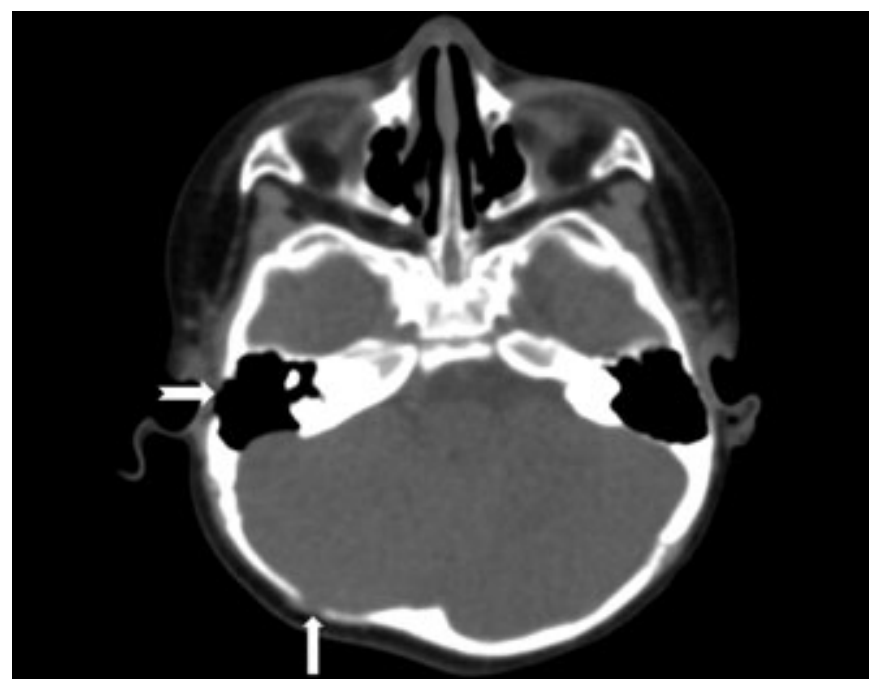

FIG. 2. An axial CT scan showed lytic lesions on the right temporal (notched arrow) and occipital bones (arrow)

imaging scan was not performed because the lesion was a bone defect and anesthesia was necessary due to the patient's age. CT (Somatom Sensation 4; Siemens, Erlangen, Germany) was performed to further investigate the lesions, and the scan showed multiple lytic lesions with smooth contours in the right parietal bone, bilateral temporal bones, bilateral frontal bones in the orbital upper-medial wall, and primarily in the occipital bone (Figure 1,2). A volume rendered CT scan provided a global view of the lytic skull lesions (Figure 3, 4). The evaluation suggested a congenital defect since the lesions were regular-shaped, there was no soft tissue component, and the adjacent calvarial bones had a normal structure and density. A joint meeting between the departments of general pediatrics, hematology-oncology, neurosurgery, and radiology concluded that the findings were consistent with an enlarged

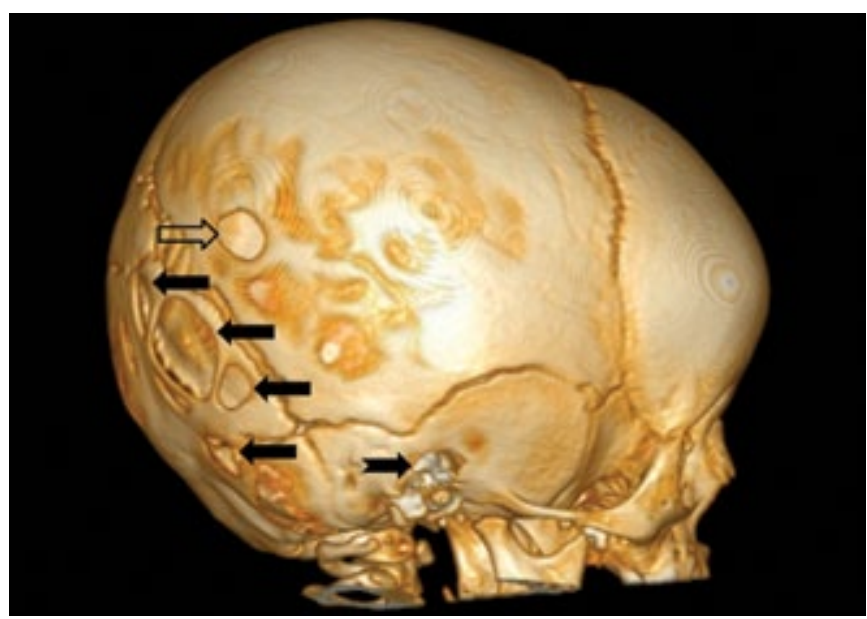

FIG. 3. A volume rendered CT scan showed multiple lytic lesions with smooth contours in the right parietal bone (open arrow), right temporal bone (notched arrow), and right side of occipital bone (arrows)

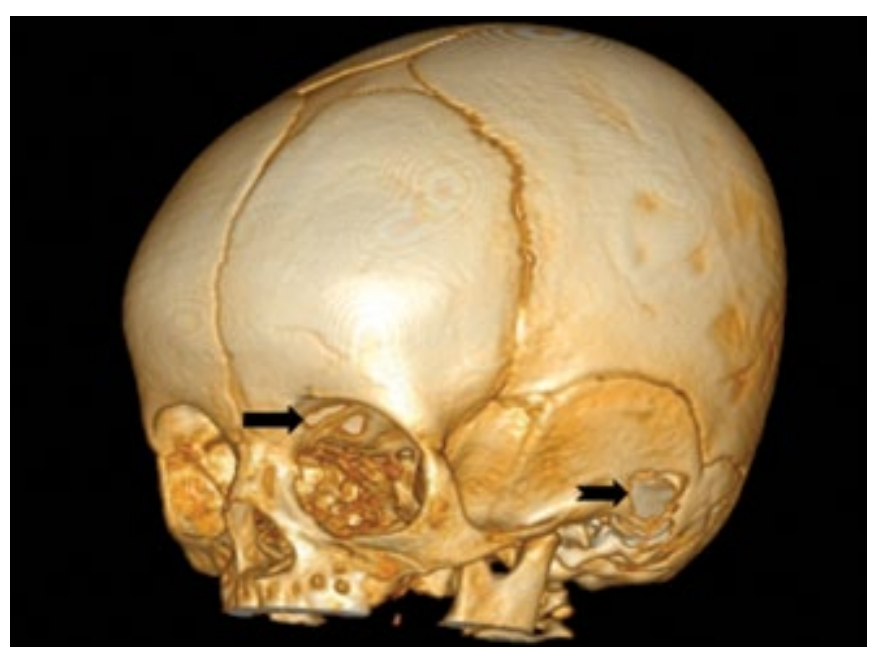

FIG. 4. A volume rendered CT scan showed multiple lytic lesions with smooth contours in the left temporal bone (notched arrow) and left frontal bone in the orbital upper-medial wall (arrow)

parietal foramina variant and elected to monitor the patient. Ultrasound follow-up was performed to avoid radiation from CT examination (Figure 5). No marked change in the lesions was observed during a three-year follow-up period.

\section{DISCUSSION}

A normal calvarium is formed by intramembranous ossification, and parietal bones begin to ossify during gestational weeks 7-8 (6). While enlarged parietal foramina are the result of impaired prenatal ossification, which is normally complete by gestational week 20 , they can also develop in the postnatal period $(4,7)$. It has been reported that foramina can shrink in size with advancing age and that there is marked intrafamilial heterogeneity (8). 


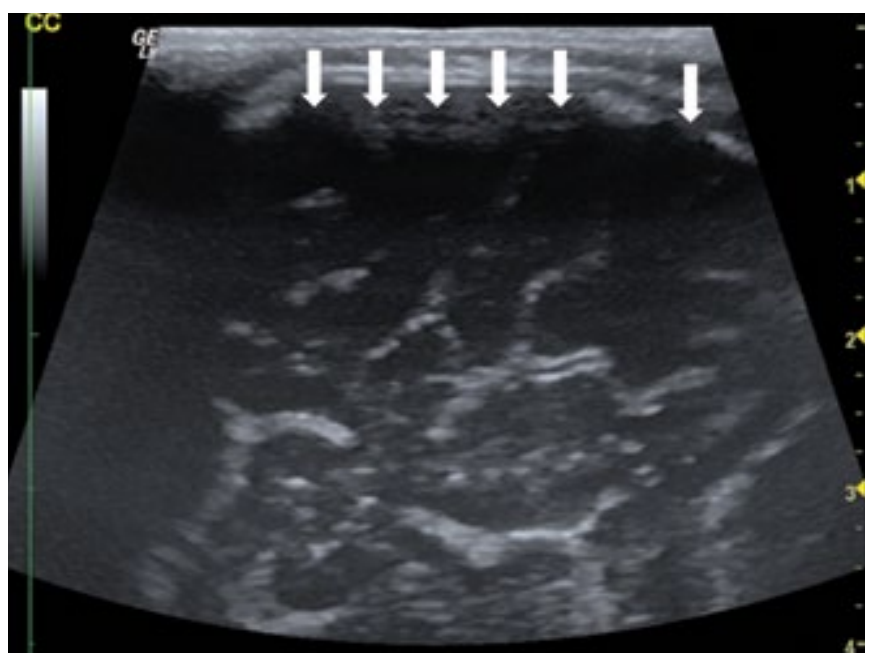

FIG. 5. An ultrasound image showed the bone defects (arrows) and provided an acoustic window to visualise the brain structures

Parietal bones are a common site of cranial defects (3). While enlarged parietal foramina are generally the result of delayed or inadequate ossification of the parietal bone, they are also known to be a normal variant of calvarial development $(4,5,8)$. This defect can be isolated or concomitant with craniofacial dysostosis, microcephaly, eye and ear abnormalities, mental retardation, seizures, vascular anomalies, and cortical defects, as well as cleidocranial dysplasia and Rubinstein-Taybi syndrome $(4,7-9)$. Our patient had no concomitant disorders.

Enlarged parietal foramina have autosomal dominant inheritance and are caused by mutations in genes MSX2 (on chromosome 5) and ALX4 (on chromosome 11) $(4,7,8)$. No clinical differences are seen in cases with these two mutations (7). They can be diagnosed with genetic testing, especially in cases with known familial mutations (7).

Differential diagnosis of cranial bone defects must be made with care. Normal parietal foramina are generally bilateral and symmetrical in the posterior parietal area, providing passage to the emissary veins connecting the occipital veins to the superior sagittal sinus, and the anastomoses to the middle meningeal and occipital arteries $(3,4)$. Lacunar skull is a dysplasia of the membranous part of the calvarium that appears as well-defined lucencies corresponding to nonossified fibrous bone. It typically resolves at six months of age (5). Enlarged parietal foramina can be separated from meningoceles, encephaloceles, and dermoid cysts based on their features, such as whole bone defects, their typical location, bilaterality, proximity to the midline, and presence of sutures connecting the defects (3). They must be radiologically differentiated from eosinophilic granuloma, epidermoid cysts, metastasis, multiple myelomas, fibrous dysplasia, osteoporosis circumscripta, and bone cysts (10). Eosinophilic granuloma is characterised by bony sequestrum and the rarely seen periostal reaction. Epidermoid cysts are lytic, have a sclerotic border and calcification of contents, and lead to remodelling and expansion of the outer and inner tables. Metastases are generally accompanied by soft tissue masses, while multiple myeloma lesions can also be found in other parts of the body (1).

Calvarial foramina tend to be multiple, but are limited to $3-4$. The presence of over six defects remote from the midline is suggestive of malignancy (2). While our patient had multiple lesions located remote from the midline, they were not malignant. A study describing a patient with foramina in the parietal, occipital, and frontal bones suggested that the smaller the infant, the more pronounced the cranial defects (3). While the frontal defect in that study was on the forehead, it was in the bilateral upper-medial orbital walls in our case. Similar to that study, our patient also had multiple bilateral temporal foramina.

To the best of our knowledge, multiple occipital, parietal, temporal, and frontal foramina have not been reported in the literature before. In patients presenting with calvarial defects, attention must be paid to the features and location of the defects. Enlarged parietal foramina should be considered in the differential diagnosis once hyperparathyroidism, osteoporosis circumcripta, hemangioma, eosinophilic granuloma, epidermoid cyst, and metastasis are ruled out.

Ethics Committee Approval: N/A.

Informed Consent: Written informed consent was obtained from the patient's parents for the publication of this case report and any accompanying images.

Peer-review: Externally peer-reviewed.

Author contributions: Concept - E.Y., A.Y., E.Y.; Design - E.Y., E.Y.; Supervision - E.Y.; Resource - E.Y., E.Y.; Materials - A.Y., O.B.E., M.P., E.Y.; Data Collection\&/or Processing - A.Y., O.B.E., M.P., E.Y.; Literature Search - E.Y.; Writing - E.Y., A.Y.; Critical Reviews - E.Y., E.Y.

Conflict of Interest: No conflict of interest was declared by the authors.

Financial Disclosure: The authors declared that this study has received no financial support.

\section{REFERENCES}

1. Arana E, Martí-Bonmatí L. CT and MR imaging of focal calvarial lesions. AJR Am J Roentgenol 1999;172:1683-8.[CrossRef]

2. Thomas JE, Baker HL Jr. Assessment of roentgenographic lucencies of the skull: a systematic approach. Neurology 1975;25:99-106.[CrossRef]

3. Lodge T. Developmental defects in the cranial vault. $\mathrm{Br} J$ Radiol 1975;48:421-34.[CrossRef] 
4. Wu XJ, Xing S, Trinkaus E. An enlarged parietal foramen in the late archaic Xujiayao 11 neurocranium from Northern China, and rare anomalies among Pleistocene Homo. PLoS One 2013;8:e59587. [CrossRef]

5. Glass RB, Fernbach SK, Norton KI, Choi PS, Naidich TP. The infant skull: a vault of information. Radiographics 2004;24:507-22. [CrossRef]

6. Fink AM, Maixner W. Enlarged parietal formina: MR imaging features in the fetus and neonate. Am J Neuroradiol 2006;27:1379-81.

7. Currarino G. Normal variants and congenital anomalies in the region of the obelion. AJR Am J Roentgenol 1976;127:487-94. [CrossRef]
8. Valente M, Valentine KD, Sugayama SSM, Kim CA. Malformation of cortical and vascular development in one family with parietal foramina determined by an ALX4 homeobox gene mutation. AJNR Am J Neuroradiol 2004;25:1836-9.

9. Chung HY, Uster-Friedberg T, Pentaz S, Blaser S, Murphy K, Chitayat D. Enlarged parietal foramina: findings on prenatal ultrasound and magnetic resonance imaging. Ultrasound Obstet Gynecol 2010;36:521-2. [CrossRef]

10. Nashold BS Jr, Netsky MG. Foramina, fenestrae, and thinness of parietal bone. J Neuropathol Exp Neurol 1959;18:432-41. [CrossRef] 